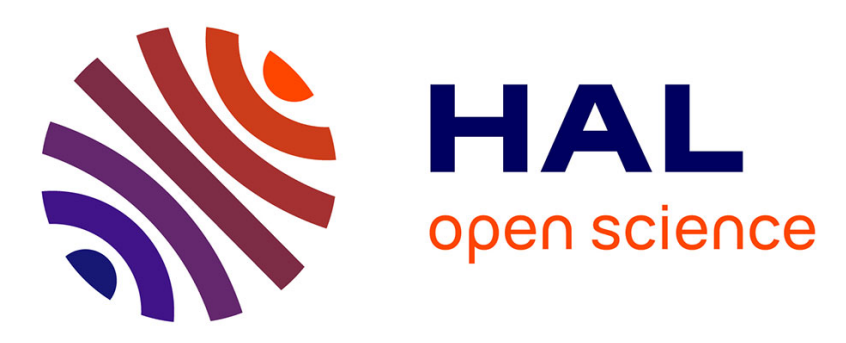

\title{
On the distribution of the summands of partitions in residue classes
}

\author{
Cécile Dartyge, Andras Sarkozy, Mihaly Szalay
}

\section{To cite this version:}

Cécile Dartyge, Andras Sarkozy, Mihaly Szalay. On the distribution of the summands of partitions in residue classes. Acta Mathematica Hungarica, 2005, 109 (3), pp.215-237. 10.1007/s10474-005-0242-7 . hal-00091859

\section{HAL Id: hal-00091859 \\ https://hal.science/hal-00091859}

Submitted on 7 Sep 2006

HAL is a multi-disciplinary open access archive for the deposit and dissemination of scientific research documents, whether they are published or not. The documents may come from teaching and research institutions in France or abroad, or from public or private research centers.
L'archive ouverte pluridisciplinaire HAL, est destinée au dépôt et à la diffusion de documents scientifiques de niveau recherche, publiés ou non, émanant des établissements d'enseignement et de recherche français ou étrangers, des laboratoires publics ou privés. 


\title{
ON THE DISTRIBUTION OF THE SUMMANDS OF PARTITIONS IN RESIDUE CLASSES
}

\author{
BY \\ CÉcile Dartyge, András SÁrközy \\ AND Mihály Szalay*
}

\begin{abstract}
It is proved that the summands of almost all partitions of $n$ are well-distributed modulo $d$ for $d$ up to $d=n^{1 / 2-\varepsilon}$.
\end{abstract}

\section{INTRODUCTION AND THE RESULTS}

The Erdős-Lehner Theorem [3] asserts that almost all the $p(n)$ unrestricted partitions of $n$ contain

$$
(1+o(1)) \frac{\sqrt{6 n}}{2 \pi} \log n
$$

parts (and the same expression approximates the maximal summand in almost all partitions of $n$ ).

In [1] and [2] Dartyge and Sárközy studied the distribution of the summands of (unrestricted) partitions in residue classes and, in particular, they showed that if $d \in \mathbb{N}$ is fixed and $n \rightarrow+\infty$, then the summands of almost all partitions of $n$ are well-distributed modulo $d$. Moreover, they applied this result to study the rate of the square-free summands to all summands in a "random" partition of $n$. (See [4] and [6] for further related results.)

If we also want to study deeper arithmetic properties of random partitions, then we have to go further, and we also have to study the distribution of the summands modulo $d$ for $d$ tending to infinity possibly fast in terms of $n$. In [1] and [2] the crucial tool was a classical theorem of Meinardus, which cannot be used in the case $d \rightarrow+\infty$. Instead, we will use here a probabilistic argument which will enable us to show that the summands themselves are well-distributed modulo $d$ for $d$ up to $d=n^{1 / 2-\varepsilon}$, and the sums of the summands belonging to the different modulo $d$ residue classes are also nearly equal for $d$ up to $d=n^{1 / 6}$. (We remark that the saddle point method also could be used and, indeed, with a slightly more work some of our error terms could be improved upon. However, for our purposes it suffices to use the more elementary approach presented here. As about the bound $n^{1 / 6}$, it is certainly not sharp. E.g., our proof also yields that each sum in question

1991 Mathematics Subject Classification. 11P82.

Key words and phrases. Partitions, residue classes.

* Research partially supported by the Hungarian National Foundation for Scientific Research, Grant T043623 and by French-Hungarian EGIDE-OMKFHÁ exchange program Balaton F-2/03. 
is $(1+o(1)) n / d$ for $d=o\left(n^{1 / 4}\right)$.) Recall that in a "random" partition of $n$ the $O(1)$ summands occur with frequency of order of magnitude $\sqrt{n}$. This fact explains certain conditions in the results (in particular, in Corollary 1) below.

Consider a general unrestricted partition of the positive integer $n$ :

$$
\Pi=\Pi(n)=\left\{\begin{array}{c}
\lambda_{1}+\lambda_{2}+\cdots+\lambda_{m}=n, \lambda_{1} \geqq \cdots \geqq \lambda_{m}(\geqq 1) \\
\lambda_{\mu} \text { 's integers, } m=m(\Pi)
\end{array}\right\} .
$$

Let $1 \leqq r \leqq d, \Lambda \geqq 1$ be integers, and in $\Pi(n)$ we investigate the number [resp. the sum] of summands $\equiv r(\bmod d)$ and $\geqq \Lambda$. We define $\Lambda^{\prime}=\left\lceil\frac{\Lambda-r}{d}\right\rceil$. (Here, $\Lambda^{\prime} \geqq 0 ; \Lambda^{\prime}=0 \Leftrightarrow 1 \leqq \Lambda \leqq r$.) As a bound for $\Lambda$ we shall use the double of the value of the main term in the Erdős-Lehner Theorem.

THEOREM 1. Let $\omega(n) \nearrow \infty$ arbitrarily slowly, $d \leqq \sqrt{n}, \Lambda \leqq \frac{\sqrt{6}}{\pi} \sqrt{n} \log n$. Then, for all but $p(n) / \omega(n)$ partitions of $n$, we have

$$
\begin{aligned}
\sum_{\substack{\mu \\
\lambda_{\mu} \equiv r(d) \\
\lambda_{\mu} \geqq \Lambda}} 1= & \frac{\sqrt{6 n}}{\pi d} \log \frac{1}{1-\exp \left(-\frac{\pi\left(r+\left(\Lambda^{\prime}+1\right) d\right)}{\sqrt{6 n}}\right)}+ \\
& +O\left(\sqrt{\frac{\sqrt{n} \omega(n)}{\left(\exp \left(\frac{\pi\left(r+\Lambda^{\prime} d\right)}{\sqrt{6 n}}\right)-1\right) \cdot \min \left(d, r+\Lambda^{\prime} d\right)}}\right) .
\end{aligned}
$$

COROLLARY 1 OF THEOREM 1 (with $\Lambda=r$ ). Let $0<\varepsilon<\frac{1}{2}$ (fixed), $1 \leqq d \leqq n^{\frac{1}{2}-\varepsilon}, \omega(n) \nearrow \infty, \frac{\omega(n)}{\log n} d \leqq r \leqq d$. Then, for all but $p(n) / \omega(n)$ partitions of $n$, we have

$$
\sum_{\substack{\mu \\ \lambda_{\mu} \equiv r(d)}} 1=(1+o(1)) \frac{\sqrt{6 n}}{\pi d} \log \left(\frac{\sqrt{n}}{d}\right), \quad \text { as } n \rightarrow \infty .
$$

COROLLARY 2 OF THEOREM 1 (with $\Lambda=d+1$ ). Let $0<\varepsilon<\frac{1}{2}$ (fixed), $1 \leqq d \leqq n^{\frac{1}{2}-\varepsilon}$. Then, for almost all partitions of $n$,

$$
\sum_{\substack{\mu \\ \lambda_{\mu} \equiv r(d) \\ \lambda_{\mu}>r}} 1=(1+o(1)) \frac{\sqrt{6 n}}{\pi d} \log \left(\frac{\sqrt{n}}{d}\right), \quad \text { as } n \rightarrow \infty .
$$

THEOREM 2. Let $\omega(n) \nearrow \infty$ arbitrarily slowly, $d \leqq \sqrt[6]{n}, \Lambda \leqq \frac{\sqrt{6}}{\pi} \sqrt{n} \log n$. Then, for all but $p(n) / \omega(n)$ partitions of $n$, we have

$$
\sum_{\substack{\mu \\ \lambda_{\mu} \equiv r(d) \\ \lambda_{\mu} \geqq \Lambda}} \lambda_{\mu}=\frac{n}{d}+O\left(\frac{\sqrt{n}}{d}\left(r+\Lambda^{\prime} d\right) \log n\right)+O\left(\frac{n^{3 / 4} \log n}{\sqrt{d}} \sqrt{\omega(n)}\right) .
$$


COROLLARY OF THEOREM 2 (with $\Lambda=r$ ). Let $\omega(n) \nearrow \infty$ arbitrarily slowly, and $d \leqq n^{1 / 6}$. Then, for all but $p(n) / \omega(n)$ partitions of $n$, we have

$$
\sum_{\substack{\mu \\ \lambda_{\mu} \equiv r(d)}} \lambda_{\mu}=\frac{n}{d}+O\left(\frac{n^{3 / 4} \log n}{\sqrt{d}} \sqrt{\omega(n)}\right) .
$$

\section{A PROBABILISTIC APPROACH}

For $1 \leqq r \leqq d, \Lambda \geqq 1$ (integers), let

$$
S_{0}(n, \Pi, \Lambda ; d, r) \stackrel{\text { def }}{=} \sum_{\substack{\mu \\ \lambda_{\mu} \equiv r(d) \\ \lambda_{\mu} \geqq \Lambda}} 1
$$

and

$$
S_{1}(n, \Pi, \Lambda ; d, r) \stackrel{\text { def }}{=} \sum_{\substack{\mu \\ \lambda_{\mu} \equiv r(d) \\ \lambda_{\mu} \geqq \Lambda}} \lambda_{\mu} .
$$

We investigate $S_{0}(n, \Pi, \Lambda ; d, r)$ and $S_{1}(n, \Pi, \Lambda ; d, r)$ together as

$$
S_{\alpha}(n, \Pi, \Lambda ; d, r)=\sum_{\substack{\mu \\ \lambda_{\mu} \equiv r(d) \\ \lambda_{\mu} \geqq \Lambda}} \lambda_{\mu}^{\alpha} .
$$

Let us consider the random field consisting of all possible choices of partitions of $n$ with equal probability. For $\alpha \in\{0,1\}$, let $\xi_{n, \alpha}$ denote a random variable which assigns the nonnegative integer $S_{\alpha}(n, \Pi, \Lambda ; d, r)$ to a partition $\Pi(n)$.

To prove Theorems 1 and 2 we will use the Chebyshev inequality. We will need estimates for the mean value $M\left(\xi_{n, \alpha}\right)$ and the variance $D^{2}\left(\xi_{n, \alpha}\right)$ of $\xi_{n, \alpha}$.

In the next paragraphs we will prove the following lemmas:

LEMMA 1. For $n \geqq n_{0}, \Lambda \leqq \frac{\sqrt{6}}{\pi} \sqrt{n} \log n, \Lambda^{\prime}=\left\lceil\frac{\Lambda-r}{d}\right\rceil$, and $d \leqq n$, we have

$$
\begin{aligned}
M\left(\xi_{n, 0}\right)= & \left(1+O\left(\frac{\log ^{2} n}{\sqrt{n}}\right)\right)\left\{\frac{\sqrt{6 n}}{\pi d} \log \frac{1}{1-\exp \left(-\frac{\pi}{\sqrt{6 n}}\left(r+\left(\Lambda^{\prime}+1\right) d\right)\right)}+\right. \\
& \left.+\frac{1}{\exp \left(\frac{\pi}{\sqrt{6 n}}\left(r+\Lambda^{\prime} d\right)\right)-1}\right\}+ \\
& +O(1) \frac{1}{\exp \left(\frac{\pi}{\sqrt{6 n}}\left(r+\left(\Lambda^{\prime}+1\right) d\right)\right)-1}+O\left(n^{-3}\right)
\end{aligned}
$$


and

$$
\begin{gathered}
M\left(\xi_{n, 1}\right)= \\
=\left(1+O\left(\frac{\log ^{2} n}{\sqrt{n}}\right)\right)\left\{\frac{n}{d}-\frac{6 n}{\pi^{2} d} \sum_{t=1}^{\infty} \frac{1}{t^{2}}\left(1-\exp \left(-\frac{\pi\left(r+\Lambda^{\prime} d\right) t}{\sqrt{6 n}}\right)\right)+\right. \\
\left.+\left(r+\Lambda^{\prime} d\right) \frac{\sqrt{6 n}}{\pi d} \log \frac{1}{1-\exp \left(-\frac{\pi\left(r+\Lambda^{\prime} d\right)}{\sqrt{6 n}}\right)}+\frac{O\left(\left(\Lambda^{\prime}+1\right) d\right)}{\exp \left(\frac{\pi\left(r+\Lambda^{\prime} d\right)}{\sqrt{6 n}}\right)-1}\right\}+O\left(n^{-3}\right) .
\end{gathered}
$$

LEMMA 2. (i) For $n \geqq n_{0}, d \leqq \sqrt{n}, \Lambda \leqq \frac{\sqrt{6}}{\pi} \sqrt{n} \log n$, and $\Lambda^{\prime}=\left\lceil\frac{\Lambda-r}{d}\right\rceil$, we have

$$
D^{2}\left(\xi_{n, 0}\right)=O\left(\frac{\sqrt{n}}{\left(\exp \left(\frac{\pi\left(r+\Lambda^{\prime} d\right)}{\sqrt{6 n}}\right)-1\right) \cdot \min \left(d, r+\Lambda^{\prime} d\right)}\right) .
$$

(ii) For $n \geqq n_{0}, d \leqq n^{5 / 12}$ and $\Lambda \leqq \frac{\sqrt{6}}{\pi} \sqrt{n} \log n$,

$$
D^{2}\left(\xi_{n, 1}\right)=O\left(\frac{n^{3 / 2} \log ^{2} n}{d}\right) .
$$

In the last paragraph, we will use these two lemmas to complete the proofs of Theorems 1 and 2.

\section{A CONSEQUENCE OF A FORMULA OF HARDY AND RAMANUJAN}

The proofs of Lemmas 1 and 2 rely on a precise estimate of the rate $\frac{p(n-t)}{p(n)}$. By [5] we have

$$
p(n)=\frac{\exp \left(\frac{2 \pi}{\sqrt{6}} \sqrt{n-\frac{1}{24}}\right)}{4\left(n-\frac{1}{24}\right) \sqrt{3}}\left\{1-\frac{\sqrt{6}}{2 \pi \sqrt{n-\frac{1}{24}}}\right\}+O\left(\exp \left(0.51 \cdot \frac{2 \pi}{\sqrt{6}} \sqrt{n}\right)\right) .
$$

This implies that

$$
p(n)=\frac{1}{4 n \sqrt{3}}\left\{1-\left(\frac{\sqrt{6}}{2 \pi}+\frac{\pi}{24 \sqrt{6}}\right) \frac{1}{\sqrt{n}}+O\left(\frac{1}{n}\right)\right\} \exp \left(\frac{2 \pi}{\sqrt{6}} \sqrt{n}\right) .
$$

LEMMA 3. For $n \geqq 2$ and $1 \leqq t \leqq n^{5 / 8}$, we have

$$
\begin{gathered}
\frac{p(n-t)}{p(n)}= \\
=\left(1+O\left(n^{-3 / 4}\right)\right)\left(1+\frac{t}{n}-\frac{\pi t^{2}}{4 n \sqrt{6 n}}-\frac{3 \pi t^{3}}{8 n^{2} \sqrt{6 n}}+\frac{\pi^{2} t^{4}}{192 n^{3}}\right) \exp \left(-\frac{\pi t}{\sqrt{6 n}}\right) .
\end{gathered}
$$


Proof. With $c=\frac{\sqrt{6}}{2 \pi}+\frac{\pi}{24 \sqrt{6}}$,

$$
\begin{aligned}
& \frac{p(n-t)}{p(n)}= \\
& =\frac{n}{n-t} \cdot \frac{1-\frac{c}{\sqrt{n-t}}+O\left(\frac{1}{n}\right)}{1-\frac{c}{\sqrt{n}}+O\left(\frac{1}{n}\right)} \exp \left(-\frac{2 \pi}{\sqrt{6}}(\sqrt{n}-\sqrt{n-t})\right)= \\
& =\left(1+\frac{t}{n}+O\left(n^{-3 / 4}\right)\right) \frac{1-\frac{c}{\sqrt{n}}+O\left(n^{-3 / 4}\right)}{1-\frac{c}{\sqrt{n}}+O\left(\frac{1}{n}\right)} \exp \left(-\frac{2 \pi}{\sqrt{6}} \cdot \frac{t}{\sqrt{n}+\sqrt{n-t}}\right)= \\
& =\left(1+O\left(n^{-3 / 4}\right)\right)\left(1+\frac{t}{n}\right) \exp \left(-\frac{\pi t}{\sqrt{6 n}}-\frac{\pi t^{2}}{4 n \sqrt{6 n}}-\right. \\
& \left.-\frac{\pi t^{2}}{\sqrt{6 n}}\left\{\frac{1}{(\sqrt{n}+\sqrt{n-t})^{2}}-\frac{1}{4 n}\right\}\right)= \\
& =\left(1+O\left(n^{-3 / 4}\right)\right)\left(1+\frac{t}{n}\right) \exp \left(-\frac{\pi t}{\sqrt{6 n}}-\frac{\pi t^{2}}{4 n \sqrt{6 n}}-\frac{\pi t^{3}}{8 n^{2} \sqrt{6 n}}+O\left(\frac{t^{4}}{n^{7 / 2}}\right)\right)= \\
& =\left(1+O\left(n^{-3 / 4}\right)\right)\left(1+\frac{t}{n}\right) \exp \left(-\frac{\pi t}{\sqrt{6 n}}\right)\left\{1-\frac{\pi t^{2}}{4 n \sqrt{6 n}}+\frac{1}{2} \frac{\pi^{2} t^{4}}{16 n^{2} 6 n}\right\} \text {. } \\
& \cdot\left\{1-\frac{\pi t^{3}}{8 n^{2} \sqrt{6 n}}\right\}= \\
& =\left(1+O\left(n^{-3 / 4}\right)\right)\left(1+\frac{t}{n}\right)\left(1-\frac{\pi t^{2}}{4 n \sqrt{6 n}}+\frac{1}{2} \frac{\pi^{2} t^{4}}{16 n^{2} 6 n}-\frac{\pi t^{3}}{8 n^{2} \sqrt{6 n}}\right) . \\
& \cdot \exp \left(-\frac{\pi t}{\sqrt{6 n}}\right)= \\
& =\left(1+O\left(n^{-3 / 4}\right)\right)\left(1+\frac{t}{n}-\frac{\pi t^{2}}{4 n \sqrt{6 n}}-\frac{3 \pi t^{3}}{8 n^{2} \sqrt{6 n}}+\frac{\pi^{2} t^{4}}{192 n^{3}}\right) \exp \left(-\frac{\pi t}{\sqrt{6 n}}\right) \text {. }
\end{aligned}
$$

4. ESTIMATIONS OF THE MEAN VALUE: PROOF OF LEMMA 1

We start from

$$
S_{\alpha}(n, \Pi, \Lambda ; d, r)=\sum_{\substack{\mu \\ \lambda_{\mu} \equiv r(d) \\ \lambda_{\mu} \geqq \Lambda}} \lambda_{\mu}^{\alpha}=\sum_{k=\Lambda^{\prime}}^{n^{\prime}}(r+k d)^{\alpha} \cdot \operatorname{mult}_{i n \Pi(n)}(r+k d)
$$

where $\Lambda^{\prime}=\left\lceil\frac{\Lambda-r}{d}\right\rceil, n^{\prime}=\left\lfloor\frac{n-r}{d}\right\rfloor$.

Let $s=\left\lfloor 6 \frac{\sqrt{6}}{\pi} \sqrt{n} \log n\right\rfloor+1($ and $p(0) \doteq 1)$.

$$
\begin{aligned}
M\left(\xi_{n, \alpha}\right) & =\frac{1}{p(n)} \sum_{\Pi(n)} S_{\alpha}(n, \Pi, \Lambda ; d, r)= \\
& =\frac{1}{p(n)} \sum_{\Pi(n)} \sum_{k=\Lambda^{\prime}}^{n^{\prime}}(r+k d)^{\alpha} \operatorname{mult}_{i n \Pi(n)}(r+k d)=
\end{aligned}
$$




$$
\begin{aligned}
& =\frac{1}{p(n)} \sum_{k=\Lambda^{\prime}}^{n^{\prime}}(r+k d)^{\alpha} \sum_{\Pi(n)} \operatorname{mult}_{i n \Pi(n)}(r+k d)= \\
& =\frac{1}{p(n)} \sum_{k=\Lambda^{\prime}}^{n^{\prime}}(r+k d)^{\alpha} \sum_{t=1}^{\left\lfloor\frac{n}{r+k d}\right\rfloor} p(n-(r+k d) t)= \\
& =\sum_{\substack{\Lambda^{\prime} \leqq k \leqq n^{\prime} \\
1 \leqq t<<\bar{s} \\
1=k+k d}}(r+k d)^{\alpha} \frac{p(n-(r+k d) t)}{p(n)}+ \\
& +\sum_{k=\Lambda^{\prime}}^{n^{\prime}}(r+k d)^{\alpha} \sum_{\substack{1 \leqq t \leqq \frac{n}{r+k d} \\
(r+k d) t \geqq s}} \frac{O(p(n-s))}{p(n)} \text { by } \stackrel{\text { Lemma }}{=} \\
& =\sum_{\Lambda^{\prime} \leqq k \leqq n^{\prime}}(r+k d)^{\alpha}\left(1+O\left(n^{-3 / 4}\right)\right)\left\{1+\frac{(r+k d) t}{n}-\frac{\pi((r+k d) t)^{2}}{4 n \sqrt{6 n}}-\right. \\
& 1 \leqq t<\frac{s}{r+k d} \\
& \left.-\frac{3 \pi((r+k d) t)^{3}}{8 n^{2} \sqrt{6 n}}+\frac{\pi^{2}((r+k d) t)^{4}}{192 n^{3}}\right\} \exp \left(-\frac{\pi}{\sqrt{6 n}}(r+k d) t\right)+ \\
& +O(n) \cdot n^{\alpha} \cdot n \cdot O\left(n^{-6}\right)= \\
& =\left(1+O\left(n^{-3 / 4}\right)\right) \sum_{k \geqq \Lambda^{\prime}}(r+k d)^{\alpha}\left\{1+\frac{(r+k d) t}{n}-\frac{\pi((r+k d) t)^{2}}{4 n \sqrt{6 n}}\right\} \text {. } \\
& \begin{array}{c}
t \geqq 1 \\
(r+k d) t<s
\end{array} \\
& \cdot \exp \left(-\frac{\pi}{\sqrt{6 n}}(r+k d) t\right)+O\left(n^{-3}\right) \quad \text { if } n \text { is large enough }
\end{aligned}
$$

since

$$
\frac{s^{3}}{n^{2} \sqrt{n}}=O\left(\frac{\log ^{3} n}{n}\right), \quad \frac{s^{4}}{n^{3}}=O\left(\frac{\log ^{4} n}{n}\right), \quad \frac{s}{n}=o(1), \quad \frac{s^{2}}{n^{3 / 2}}=o(1),
$$

moreover, $r+k d<\frac{s}{t}$ implies that $k \leqq n^{\prime}$ if $n \geqq n_{0}$. Thus we have proved the following

LEMMA 4. For $n \geqq n_{0}, \Lambda \leqq \frac{\sqrt{6}}{\pi} \sqrt{n} \log n, d \leqq n$, and $s=\left\lfloor 6 \frac{\sqrt{6}}{\pi} \sqrt{n} \log n\right\rfloor+1$, we have

$$
\begin{gathered}
M\left(\xi_{n, 0}\right)= \\
=\left(1+O\left(n^{-3 / 4}\right)\right) \sum_{\substack{k \geqq \Lambda^{\prime} \\
t \geqq 1 \\
(r+k d) t<s}}\left(1+\frac{(r+k d) t}{n}-\frac{\pi((r+k d) t)^{2}}{4 n \sqrt{6 n}}\right) . \\
\cdot \exp \left(-\frac{\pi}{\sqrt{6 n}}(r+k d) t\right)+O\left(n^{-3}\right)
\end{gathered}
$$


and

$$
\begin{gathered}
M\left(\xi_{n, 1}\right)= \\
=\left(1+O\left(n^{-3 / 4}\right)\right) \sum_{\substack{k \geqq \Lambda^{\prime} \\
t \geqq 1 \\
(r+k d) t<s}}(r+k d)\left\{1+\frac{(r+k d) t}{n}-\frac{\pi((r+k d) t)^{2}}{4 n \sqrt{6 n}}\right\} . \\
\cdot \exp \left(-\frac{\pi}{\sqrt{6 n}}(r+k d) t\right)+O\left(n^{-3}\right) .
\end{gathered}
$$

Again, let $n \geqq n_{0}, \Lambda \leqq \frac{\sqrt{6}}{\pi} \sqrt{n} \log n, d \leqq n, s=\left\lfloor 6 \frac{\sqrt{6}}{\pi} \sqrt{n} \log n\right\rfloor+1$, $\alpha \in\{0,1\}$. Since

Lemma 4 yields that

$$
\frac{s}{n}=O\left(\frac{\log n}{\sqrt{n}}\right) \quad \text { and } \quad \frac{s^{2}}{n^{3 / 2}}=O\left(\frac{\log ^{2} n}{\sqrt{n}}\right),
$$

$$
\begin{aligned}
& \left(1+O\left(\frac{\log ^{2} n}{\sqrt{n}}\right)\right) \sum_{\substack{k \geqq \Lambda^{\prime} \\
t \geqq 1 \\
(r+k d) t<s}}(r+k d)^{\alpha} \exp \left(-\frac{\pi}{\sqrt{6 n}}(r+k d) t\right)+O\left(\xi^{-3}\right)= \\
= & \left(1+O\left(\frac{\log ^{2} n}{\sqrt{n}}\right)\right) \sum_{\substack{k \geqq \Lambda^{\prime} \\
t \geqq 1}}(r+k d)^{\alpha} \exp \left(-\frac{\pi}{\sqrt{6 n}}(r+k d) t\right)+ \\
& +O(1) \quad \sum_{k \geqq \Lambda^{\prime}}(r+k d)^{\alpha} \exp \left(-\frac{\pi}{\sqrt{6 n}}(r+k d) t\right)+O\left(n^{-3}\right)= \\
= & \left(1+O\left(\frac{\log ^{2} n}{\sqrt{n}}\right)\right) \sum_{\substack{k=\Lambda^{\prime} \\
t \geq 1=1}}^{\infty} \sum_{t=s}^{\infty}(r+k d)^{\alpha} \exp \left(-\frac{\pi}{\sqrt{6 n}}(r+k d) t\right)+ \\
& +O(1) \sum_{j=s}^{\infty} \exp \left(-\frac{\pi}{\sqrt{6 n}} j\right) \sum_{\substack{(r+k d) t=j \\
t \geqq 1, k \geqq \Lambda^{\prime}}}^{\infty}(r+k d)^{\alpha}+O\left(n^{-3}\right)= \\
= & \left(1+O\left(\frac{\log ^{2} n}{\sqrt{n}}\right)\right) \sum_{k=\Lambda^{\prime}}^{\infty} \sum_{t=1}^{\infty}(r+k d)^{\alpha} \exp \left(-\frac{\pi}{\sqrt{6 n}}(r+k d) t\right)+ \\
& +O(1) \sum_{j=s}^{\infty} \exp \left(-\frac{\pi}{\sqrt{6 n}} j\right) O\left(j \cdot j^{\alpha}\right)+O\left(n^{-3}\right) .
\end{aligned}
$$

Here, with $x=\exp \left(-\frac{\pi}{\sqrt{6 n}}\right)$,

$$
\begin{aligned}
& \sum_{j=s}^{\infty} j \exp \left(-\frac{\pi}{\sqrt{6 n}} j\right)=\sum_{j=s}^{\infty} j x^{j}=x\left(\sum_{j=s}^{\infty} x^{j}\right)^{\prime}=x\left(\frac{x^{s}}{1-x}\right)^{\prime}= \\
& =x \frac{s x^{s-1}(1-x)+x^{s}}{(1-x)^{2}}=\frac{s x^{s}}{1-x}+\frac{x^{s+1}}{(1-x)^{2}}=x^{s}\left(\frac{s}{1-x}+\frac{x}{(1-x)^{2}}\right)
\end{aligned}
$$


and

$$
\begin{aligned}
& \sum_{j=s}^{\infty} j^{2} \exp \left(-\frac{\pi}{\sqrt{6 n}} j\right)=\sum_{j=s}^{\infty} j^{2} x^{j}=x\left(\sum_{j=s}^{\infty} j x^{j}\right)^{\prime}= \\
& =x\left(\frac{s x^{s}}{1-x}+\frac{x^{s+1}}{(1-x)^{2}}\right)^{\prime}= \\
& =x\left(\frac{s^{2} x^{s-1}(1-x)-s \cdot x^{s} \cdot(-1)}{(1-x)^{2}}+\frac{(s+1) x^{s}(1-x)^{2}-x^{s+1} 2(1-x)(-1)}{(1-x)^{4}}\right)= \\
& =x^{s}\left(\frac{s^{2}}{1-x}+\frac{s x}{(1-x)^{2}}+\frac{(s+1) x}{(1-x)^{2}}+\frac{2 x^{2}}{(1-x)^{3}}\right)= \\
& =x^{s}\left(\frac{s^{2}}{1-x}+\frac{(2 s+1) x}{(1-x)^{2}}+\frac{2 x^{2}}{(1-x)^{3}}\right)= \\
& =x^{s}\left(O\left(n^{3 / 2} \log ^{2} n\right)+O\left(n^{3 / 2} \log n\right)+O\left(n^{3 / 2}\right)\right)= \\
& =x^{s} O\left(n^{3 / 2} \log ^{2} n\right)=O\left(n^{-6} n^{3 / 2} \log ^{2} n\right) .
\end{aligned}
$$

Consequently,

$$
\sum_{j=s}^{\infty} j^{\alpha+1} \exp \left(-\frac{\pi}{\sqrt{6 n}} j\right)=O\left(n^{-4.5} \log ^{2} n\right),
$$

and

$$
\begin{gathered}
M\left(\xi_{n, \alpha}\right)= \\
=\left(1+O\left(\frac{\log ^{2} n}{\sqrt{n}}\right)\right) \sum_{k=\Lambda^{\prime}}^{\infty} \sum_{t=1}^{\infty}(r+k d)^{\alpha} \exp \left(-\frac{\pi}{\sqrt{6 n}}(r+k d) t\right)+O\left(n^{-3}\right) .
\end{gathered}
$$

$[\alpha=0:]$

The following separation of $k=\Lambda^{\prime}$ plays a role for $\Lambda^{\prime}=0$ :

$$
\begin{aligned}
& M\left(\xi_{n, 0}\right)=\left(1+O\left(\frac{\log ^{2} n}{\sqrt{n}}\right)\right) \sum_{t=1}^{\infty} \exp \left(-\frac{\pi}{\sqrt{6 n}}\left(r+\Lambda^{\prime} d\right) t\right)+ \\
& +\left(1+O\left(\frac{\log ^{2} n}{\sqrt{n}}\right)\right) \sum_{t=1}^{\infty} \exp \left(-\frac{\pi r t}{\sqrt{6 n}}\right) \sum_{k=\Lambda^{\prime}+1}^{\infty}\left(\exp \left(-\frac{\pi d t}{\sqrt{6 n}}\right)\right)^{k}+ \\
& =\left(1+O\left(\frac{\log ^{2} n}{\sqrt{n}}\right)\right) \frac{\left.1 n^{-3}\right)=}{\exp \left(\frac{\pi}{\sqrt{6 n}}\left(r+\Lambda^{\prime} d\right)\right)-1}+O\left(n^{-3}\right)+ \\
& \quad+\left(1+O\left(\frac{\log ^{2} n}{\sqrt{n}}\right)\right) \sum_{t=1}^{\infty} \exp \left(-\frac{\pi}{\sqrt{6 n}}\left(r+\left(\Lambda^{\prime}+1\right) d\right) t\right) \frac{1}{1-\exp \left(-\frac{\pi d t}{\sqrt{6 n}}\right)} .
\end{aligned}
$$

For real $\beta>0$, we have

$$
\frac{1}{2}<\frac{1}{1-e^{-\beta}}-\frac{1}{\beta}<1
$$


since

$$
1>1+\frac{1}{e^{\beta}-1}-\frac{1}{\beta}=1-\frac{\frac{\beta}{2 !}+\frac{\beta^{2}}{3 !}+\ldots}{e^{\beta}-1}>1-\frac{\frac{\beta}{2}+\frac{\beta^{2}}{2 \cdot 2 !}+\ldots}{e^{\beta}-1}=\frac{1}{2} .
$$

Thus, by using the formula $\sum_{t=1}^{\infty} \frac{e^{-a t}}{t}=\log \frac{1}{1-e^{-a}}$ with $a=\frac{\pi}{\sqrt{6 n}}\left(r+\left(\Lambda^{\prime}+\right.\right.$ 1)d),

$$
\begin{aligned}
& M\left(\xi_{n, 0}\right)=\left(1+O\left(\frac{\log ^{2} n}{\sqrt{n}}\right)\right) \frac{1}{\exp \left(\frac{\pi}{\sqrt{6 n}}\left(r+\Lambda^{\prime} d\right)\right)-1}+O\left(n^{-3}\right)+ \\
& +\left(1+O\left(\frac{\log ^{2} n}{\sqrt{n}}\right)\right) \sum_{t=1}^{\infty} \exp \left(-\frac{\pi}{\sqrt{6 n}}\left(r+\left(\Lambda^{\prime}+1\right) d\right) t\right)\left\{\frac{\sqrt{6 n}}{\pi d t}+O(1)\right\}= \\
& =\left(1+O\left(\frac{\log ^{2} n}{\sqrt{n}}\right)\right)\left\{\frac{1}{\exp \left(\frac{\pi}{\sqrt{6 n}}\left(r+\Lambda^{\prime} d\right)\right)-1}+\right. \\
& \left.+\frac{\sqrt{6 n}}{\pi d} \log \frac{1}{1-\exp \left(-\frac{\pi}{\sqrt{6 n}}\left(r+\left(\Lambda^{\prime}+1\right) d\right)\right)}\right\}+ \\
& +O(1) \frac{1}{\exp \left(\frac{\pi}{\sqrt{6 n}}\left(r+\left(\Lambda^{\prime}+1\right) d\right)\right)-1}+O\left(n^{-3}\right) . \\
& {[\alpha=1:]} \\
& M\left(\xi_{n, 1}\right)= \\
& =\left(1+O\left(\frac{\log ^{2} n}{\sqrt{n}}\right)\right) \sum_{t=1}^{\infty} \exp \left(-\frac{\pi r t}{\sqrt{6 n}}\right) \sum_{k=\Lambda^{\prime}}^{\infty}(r+k d)^{1}\left(\exp \left(-\frac{\pi d t}{\sqrt{6 n}}\right)\right)^{k}+ \\
& +O\left(n^{-3}\right)= \\
& =\left(1+O\left(\frac{\log ^{2} n}{\sqrt{n}}\right)\right) \sum_{t=1}^{\infty} \exp \left(-\frac{\pi r t}{\sqrt{6 n}}\right)\left\{r \sum_{k=\Lambda^{\prime}}^{\infty}\left(\exp \left(-\frac{\pi d t}{\sqrt{6 n}}\right)\right)^{k}+\right. \\
& \left.+d \sum_{k=\Lambda^{\prime}}^{\infty} k\left(\exp \left(-\frac{\pi d t}{\sqrt{6 n}}\right)\right)^{k}\right\}+O\left(n^{-3}\right) \text {. }
\end{aligned}
$$

Since, for $y=\exp \left(-\frac{\pi d t}{\sqrt{6 n}}\right)$,

$$
\sum_{k=\Lambda^{\prime}}^{\infty} k y^{k}=y^{\Lambda^{\prime}}\left(\frac{\Lambda^{\prime}}{1-y}+\frac{y}{(1-y)^{2}}\right)
$$

we obtain that

$$
\begin{gathered}
M\left(\xi_{n, 1}\right)= \\
=\left(1+O\left(\frac{\log ^{2} n}{\sqrt{n}}\right)\right) \sum_{t=1}^{\infty} \exp \left(-\frac{\pi r t}{\sqrt{6 n}}\right)\left\{\frac{\exp \left(-\frac{\pi d t \Lambda^{\prime}}{\sqrt{6 n}}\right)}{1-\exp \left(-\frac{\pi d t}{\sqrt{6 n}}\right)}+\right.
\end{gathered}
$$




$$
\begin{gathered}
\left.+d \cdot \exp \left(-\frac{\pi d t \Lambda^{\prime}}{\sqrt{6 n}}\right) \cdot\left(\frac{\Lambda^{\prime}}{1-\exp \left(-\frac{\pi d t}{\sqrt{6 n}}\right)}+\frac{\exp \left(-\frac{\pi d t}{\sqrt{6 n}}\right)}{\left(1-\exp \left(-\frac{\pi d t}{\sqrt{6 n}}\right)\right)^{2}}\right)\right\}+ \\
+O\left(n^{-3}\right)= \\
=\left(1+O\left(\frac{\log ^{2} n}{\sqrt{n}}\right)\right) \sum_{t=1}^{\infty} \exp \left(-\frac{\pi\left(r+\Lambda^{\prime} d\right) t}{\sqrt{6 n}}\right)\left\{\frac{r+\Lambda^{\prime} d}{1-\exp \left(-\frac{\pi d t}{\sqrt{6 n}}\right)}+\right. \\
\left.+d \frac{\exp \left(-\frac{\pi d t}{\sqrt{6 n}}\right)}{\left(1-\exp \left(-\frac{\pi d t}{\sqrt{6 n}}\right)\right)^{2}}\right\}+O\left(n^{-3}\right) .
\end{gathered}
$$

For real $\beta>0$, we have

$$
\frac{1}{\beta}+\frac{1}{2}<\frac{1}{1-e^{-\beta}}<\frac{1}{\beta}+1 .
$$

Thus,

$$
\frac{1}{\beta}-\frac{1}{2}<\frac{1}{e^{\beta}-1}=\frac{1}{1-e^{-\beta}}-1<\frac{1}{\beta} .
$$

For $0<\beta<2$ these imply that

$$
\frac{1}{\beta^{2}}-\frac{1}{4}<\frac{1}{\left(e^{\beta}-1\right)\left(1-e^{-\beta}\right)}
$$

which is trivial for $\beta \geqq 2$. Next,

$$
\begin{aligned}
\frac{1}{\left(e^{\beta}-1\right)\left(1-e^{-\beta}\right)} & =\left(\frac{e^{\beta / 2}}{e^{\beta}-1}\right)^{2}=\left(\frac{e^{\beta / 2}}{\beta\left(1+\frac{\beta}{2 !}+\frac{\beta^{2}}{3 !}+\cdots+\frac{\beta^{k}}{(k+1) !}+\ldots\right)}\right)^{2}< \\
& <\left(\frac{e^{\beta / 2}}{\beta\left(1+\frac{\beta / 2}{1 !}+\frac{(\beta / 2)^{2}}{2 !}+\cdots+\frac{(\beta / 2)^{k}}{k !}+\ldots\right)}\right)^{2}=\frac{1}{\beta^{2}}
\end{aligned}
$$

For $\beta>0$ we obtained that

$$
\frac{1}{\beta^{2}}-\frac{1}{4}<\frac{e^{-\beta}}{\left(1-e^{-\beta}\right)^{2}}<\frac{1}{\beta^{2}} .
$$

Therefore,

$$
\begin{gathered}
M\left(\xi_{n, 1}\right)= \\
=\left(1+O\left(\frac{\log ^{2} n}{\sqrt{n}}\right)\right) \sum_{t=1}^{\infty} \exp \left(-\frac{\pi\left(r+\Lambda^{\prime} d\right) t}{\sqrt{6 n}}\right)\left\{\left(r+\Lambda^{\prime} d\right)\left(\frac{\sqrt{6 n}}{\pi d t}+O(1)\right)+\right. \\
\left.+d \cdot\left(\left(\frac{\sqrt{6 n}}{\pi d t}\right)^{2}+O(1)\right)\right\}+O\left(n^{-3}\right)=
\end{gathered}
$$




$$
\begin{aligned}
= & \left(1+O\left(\frac{\log ^{2} n}{\sqrt{n}}\right)\right)\left\{\frac{6 n}{\pi^{2} d} \sum_{t=1}^{\infty} \frac{1}{t^{2}} \exp \left(-\frac{\pi\left(r+\Lambda^{\prime} d\right) t}{\sqrt{6 n}}\right)+\right. \\
& +\left(r+\Lambda^{\prime} d\right) \frac{\sqrt{6 n}}{\pi d} \sum_{t=1}^{\infty} \frac{1}{t} \exp \left(-\frac{\pi\left(r+\Lambda^{\prime} d\right) t}{\sqrt{6 n}}\right)+ \\
& \left.+O\left(\left(\Lambda^{\prime}+1\right) d\right) \sum_{t=1}^{\infty} \exp \left(-\frac{\pi\left(r+\Lambda^{\prime} d\right) t}{\sqrt{6 n}}\right)\right\}+O\left(n^{-3}\right)= \\
= & \left(1+O\left(\frac{\log ^{2} n}{\sqrt{n}}\right)\right)\left\{\frac{n}{d}-\frac{6 n}{\pi^{2} d} \sum_{t=1}^{\infty} \frac{1}{t^{2}}\left(1-\exp \left(-\frac{\pi\left(r+\Lambda^{\prime} d\right) t}{\sqrt{6 n}}\right)\right)+\right. \\
& \left.+\left(r+\Lambda^{\prime} d\right) \frac{\sqrt{6 n}}{\pi d} \log \frac{1}{1-\exp \left(-\frac{\pi\left(r+\Lambda^{\prime} d\right)}{\sqrt{6 n}}\right)}+\frac{O\left(\left(\Lambda^{\prime}+1\right) d\right)}{\exp \left(\frac{\pi\left(r+\Lambda^{\prime} d\right)}{\sqrt{6 n}}\right)-1}\right\}+
\end{aligned}
$$

Thus we have proved our Lemma 1. Later we shall also use the following Remark 1. Here, since for $\beta \geqq 0,1-e^{-\beta} \leqq \beta$ holds,

$$
\begin{gathered}
\frac{6 n}{\pi^{2} d} \sum_{t=1}^{\infty} \frac{1}{t^{2}}\left(1-\exp \left(-\frac{\pi\left(r+\Lambda^{\prime} d\right) t}{\sqrt{6 n}}\right)\right)= \\
=\frac{6 n}{\pi^{2} d}\left(\sum_{t=1}^{n} \frac{1}{t^{2}} O\left(\frac{\left(r+\Lambda^{\prime} d\right) t}{\sqrt{n}}\right)+\sum_{t=n+1}^{\infty} \frac{1}{t^{2}} O(1)\right)= \\
=\frac{6 n}{\pi^{2} d}\left(O\left(\frac{r+\Lambda^{\prime} d}{\sqrt{n}}\right) O(\log n)+O\left(\frac{1}{n}\right)\right)=O\left(\frac{\sqrt{n}}{d}\left(r+\Lambda^{\prime} d\right) \log n\right)+ \\
+O\left(\frac{1}{d}\right)
\end{gathered}
$$

\section{PROOF OF LEMMA 2}

Since

$$
\begin{aligned}
D^{2}\left(\xi_{n, \alpha}\right) & =M\left(\left(\xi_{n, \alpha}-M\left(\xi_{n, \alpha}\right)\right)^{2}\right)= \\
& =M\left(\xi_{n, \alpha}^{2}\right)-\left(M\left(\xi_{n, \alpha}\right)\right)^{2}
\end{aligned}
$$

we shall estimate $M\left(\xi_{n, \alpha}^{2}\right)$ from above (for $\alpha \in\{0,1\}$ ).

$$
\begin{gathered}
M\left(\xi_{n, \alpha}^{2}\right)=\frac{1}{p(n)} \sum_{\Pi(n)} S_{\alpha}^{2}(n, \Pi, \Lambda ; d, r)= \\
=\frac{1}{p(n)} \sum_{\Pi(n)}\left(\sum_{k=\Lambda^{\prime}}^{n^{\prime}}(r+k d)^{\alpha} \operatorname{mult}_{i n \Pi(n)}(r+k d)\right)\left(\sum_{j=\Lambda^{\prime}}^{n^{\prime}}(r+j d)^{\alpha} \operatorname{mult}_{i n \Pi(n)}(r+j d)\right)= \\
=\frac{1}{p(n)} \sum_{k=\Lambda^{\prime}}^{n^{\prime}}(r+k d)^{\alpha} \sum_{j=\Lambda^{\prime}}^{n^{\prime}}(r+j d)^{\alpha} \sum_{\Pi(n)} \operatorname{mult}_{i n \Pi(n)}(r+k d) \cdot \operatorname{mult}_{i n \Pi(n)}(r+j d)=
\end{gathered}
$$




$$
\begin{aligned}
& =\frac{1}{p(n)} \sum_{k=\Lambda^{\prime}}^{n^{\prime}}(r+k d)^{\alpha} \sum_{j=\Lambda^{\prime}}^{n^{\prime}}(r+j d)^{\alpha} \sum_{t_{1}=1}^{n} \sum_{t_{2}=1}^{n} \sum_{\substack{\Pi(n) \\
\text { mult } \\
i n \Pi(n) \\
\text { mult } \\
i n \Pi(n)(r+k d) \geqq t_{1}}} 1= \\
& =\frac{1}{p(n)} \sum_{k=\Lambda^{\prime}}^{n^{\prime}}(r+k d)^{\alpha} \sum_{\substack{j=\Lambda^{\prime} \\
j \neq k}}^{n^{\prime}}(r+j d)^{\alpha} \sum_{\substack{t_{1}, t_{2} \geqq 1 \\
t_{1}(r+k d)+t_{2}(r+j d) \leqq n}} p\left(n-t_{1}(r+k d)-\right. \\
& \left.-t_{2}(r+j d)\right)+\frac{1}{p(n)} \sum_{k=\Lambda^{\prime}}^{n^{\prime}}(r+k d)^{2 \alpha} \sum_{\substack{t \geqq 1 \\
t(r+k d) \leqq n}}(2 t-1) p(n-t(r+k d))= \\
& =\frac{1}{p(n)} \sum_{k=\Lambda^{\prime}}^{n^{\prime}}(r+k d)^{\alpha} \sum_{j=\Lambda^{\prime}}^{n^{\prime}}(r+j d)^{\alpha} \sum_{\substack{t_{1}, t_{2} \geqq 1 \\
t_{1}(r+k d)+t_{2}(r+j d) \leqq n}} p\left(n-t_{1}(r+k d)-\right. \\
& \left.-t_{2}(r+j d)\right)+\frac{1}{p(n)} \sum_{k=\Lambda^{\prime}}^{n^{\prime}}(r+k d)^{2 \alpha} \sum_{\substack{t \geqq 1 \\
t(r+k d) \leqq n}} t \cdot p(n-t(r+k d)) .
\end{aligned}
$$

By Lemma 3, with $s=\left\lfloor 6 \frac{\sqrt{6}}{\pi} \sqrt{n} \log n\right\rfloor+1$ and $n \geqq n_{0}^{\prime}$, since

$$
\begin{gathered}
\sum_{\substack{k \geqq \Lambda^{\prime} \\
t \geq 1 \\
t(r+k d) \leqq n}} 1 \leqq \sum_{m=1}^{n} \frac{n}{m}=O(n \log n), \\
=\sum_{\substack{k, j \geqq \Lambda^{\prime} \\
t_{1}, t_{2} \geq 1 \\
t_{1}(r+k d)<s \\
t_{2}(r+j d)<s}}(r+k d)^{\alpha}(r+j d)^{\alpha} \frac{p\left(n-t_{1}(r+k d)-t_{2}(r+j d)\right)}{p(n)}+ \\
+O\left((n \log n)^{2} n^{2 \alpha} n^{-6}\right)+ \\
+\sum_{\substack{k \geq \Lambda^{\prime} \\
t \geq 1 \\
t(r+k d)<s}}(r+k d)^{2 \alpha} t \frac{p(n-t(r+k d))}{p(n)}+O\left((n \log n) n^{2 \alpha} \cdot n \cdot n^{-6}\right)= \\
=\sum_{\substack{k, j \geqq \Lambda^{\prime} \\
t_{1} t_{2} \geq 1 \\
t_{1}(r+k d)<s \\
t_{2}(r+j d)<s}}(r+k d)^{\alpha}(r+j d)^{\alpha}\left(1+O\left(n^{-3 / 4}\right)\right)\left(1+\frac{t_{1}(r+k d)+t_{2}(r+j d)}{n}-\right. \\
\left.-\frac{\pi\left(t_{1}(r+k d)+t_{2}(r+j d)\right)^{2}}{4 n \sqrt{6 n}}\right) \exp \left(-\frac{\pi\left(t_{1}(r+k d)+t_{2}(r+j d)\right)}{\sqrt{6 n}}\right)+
\end{gathered}
$$




$$
\begin{aligned}
& +O\left(n^{-2} \log ^{2} n\right)+ \\
& +\sum_{\substack{k \geqq \Lambda^{\prime} \\
t \geq 1 \\
t(r+k d)<s}}(r+k d)^{2 \alpha} t\left(1+O\left(n^{-3 / 4}\right)\right)\left(1+\frac{t(r+k d)}{n}-\right. \\
& \\
& \left.\quad-\frac{\pi(t(r+k d))^{2}}{4 n \sqrt{6 n}}\right) \exp \left(-\frac{\pi t(r+k d)}{\sqrt{6 n}}\right) .
\end{aligned}
$$

In the first multisum, for sufficiently large $n$,

$$
\begin{gathered}
1+\frac{t_{1}(r+k d)+t_{2}(r+j d)}{n}-\frac{\pi\left(t_{1}(r+k d)+t_{2}(r+j d)\right)^{2}}{4 n \sqrt{6 n}}< \\
<1+\frac{t_{1}(r+k d)}{n}+\frac{t_{2}(r+j d)}{n}-\frac{\pi\left(t_{1}(r+k d)\right)^{2}}{4 n \sqrt{6 n}-\frac{\pi\left(t_{2}(r+j d)\right)^{2}}{4 n \sqrt{6 n}}=} \\
=\left(1+\frac{t_{1}(r+k d)}{n}-\frac{\pi\left(t_{1}(r+k d)\right)^{2}}{4 n \sqrt{6 n}}\right)\left(1+\frac{t_{2}(r+j d)}{n}-\frac{\pi\left(t_{2}(r+j d)\right)^{2}}{4 n \sqrt{6 n}}\right)+ \\
+O\left(\frac{\log ^{4} n}{n}\right)= \\
=\left(1+O\left(n^{-3 / 4}\right)\right)\left(1+\frac{t_{1}(r+k d)}{n}-\frac{\pi\left(t_{1}(r+k d)\right)^{2}}{4 n \sqrt{6 n}}\right) . \\
\cdot\left(1+\frac{t_{2}(r+j d)}{n}-\frac{\pi\left(t_{2}(r+j d)\right)^{2}}{4 n \sqrt{6 n}}\right) .
\end{gathered}
$$

Consequently,

$$
\begin{gathered}
M\left(\xi_{n, \alpha}^{2}\right) \leqq \\
\leqq\left(1+O\left(n^{-3 / 4}\right)\right)\left\{\sum_{\substack{k \geqq \Lambda^{\prime} \\
t_{1} \geq 1 \\
t_{1}(r+\bar{k} d)<s}}(r+k d)^{\alpha}\left(1+\frac{t_{1}(r+k d)}{n}-\frac{\pi\left(t_{1}(r+k d)\right)^{2}}{4 n \sqrt{6 n}}\right) .\right. \\
\left.\cdot \exp \left(-\frac{\pi t_{1}(r+k d)}{\sqrt{6 n}}\right)\right\}^{2}+O\left(n^{-2} \log ^{2} n\right)+ \\
+\left(1+O\left(n^{-3 / 4}\right)\right) \sum_{\substack{k \geqq \Lambda^{\prime} \\
t \geqq 1 \\
t(r+\bar{k} d)<s}}(r+k d)^{2 \alpha} t\left(1+\frac{t(r+k d)}{n}-\frac{\pi(t(r+k d))^{2}}{4 n \sqrt{6 n}}\right) . \\
\cdot \exp \left(-\frac{\pi t(r+k d)}{\sqrt{6 n}}\right) .
\end{gathered}
$$

Remark 2. It is supposed that $d \leqq \sqrt{n}$. Then $r+\Lambda^{\prime} d \leqq \Lambda+d<s$, i.e., $1\left(r+\Lambda^{\prime} d\right)<s$ and the error term in the term with $k=\Lambda^{\prime}$ and $t=1$ in the last sum is

$$
O\left(n^{-3 / 4}\right)\left(r+\Lambda^{\prime} d\right)^{2 \alpha} \exp \left(-\frac{\pi\left(r+\Lambda^{\prime} d\right)}{\sqrt{6 n}}\right)
$$


which majorizes the error term $O\left(n^{-2} \log ^{2} n\right)$ since

$$
\left(r+\Lambda^{\prime} d\right)^{2 \alpha} \exp \left(-\frac{\pi\left(r+\Lambda^{\prime} d\right)}{\sqrt{6 n}}\right) \geqq \exp \left(-\frac{\pi(\Lambda+d)}{\sqrt{6 n}}\right)>\frac{1}{e^{2} n} .
$$

Therefore, the error term $O\left(n^{-2} \log ^{2} n\right)$ can be dropped. The same argument can be applied to the error terms $O\left(n^{-3}\right)$ in Lemma 4. Consequently, each error term $O\left(n^{-3}\right)$ of Lemma 1 can be dropped for $d \leqq \sqrt{n}$.

In this way, for $\Lambda \leqq \frac{\sqrt{6}}{\pi} \sqrt{n} \log n, d \leqq \sqrt{n}$ and $n \geqq n_{0}^{\prime \prime}$, we obtain that

$$
\begin{aligned}
& M\left(\xi_{n, \alpha}^{2}\right) \leqq\left(1+O\left(n^{-3 / 4}\right)\right) M^{2}\left(\xi_{n, \alpha}\right)+ \\
& +\left(1+O\left(\frac{\log ^{2} n}{\sqrt{n}}\right)\right) \sum_{\substack{k \geqq \Lambda^{\prime} \\
t \geqq 1 \\
t(r+\bar{k} d)<s}}(r+k d)^{2 \alpha} t \exp \left(-\frac{\pi t(r+k d)}{\sqrt{6 n}}\right) .
\end{aligned}
$$

Finally,

$$
\begin{aligned}
& D^{2}\left(\xi_{n, \alpha}\right)=M\left(\xi_{n, \alpha}^{2}\right)-M^{2}\left(\xi_{n, \alpha}\right) \leqq O\left(n^{-3 / 4}\right) M^{2}\left(\xi_{n, \alpha}\right)+ \\
& \quad+\left(1+O\left(\frac{\log ^{2} n}{\sqrt{n}}\right)\right) \sum_{\substack{k \geqq \Lambda^{\prime} \\
t \geqq 1 \\
t(r+\bar{k} d)<s}}(r+k d)^{2 \alpha} t \exp \left(-\frac{\pi t(r+k d)}{\sqrt{6 n}}\right) .
\end{aligned}
$$

$[\alpha=0:]$

$$
\begin{aligned}
D^{2}\left(\xi_{n, 0}\right) \leqq O\left(n^{-3 / 4}\right) M^{2}\left(\xi_{n, 0}\right)+ \\
\quad+\left(1+O\left(\frac{\log ^{2} n}{\sqrt{n}}\right)\right) \sum_{k=\Lambda^{\prime}}^{\infty} \sum_{t=1}^{\infty} t \exp \left(-\frac{\pi t(r+k d)}{\sqrt{6 n}}\right)= \\
=O\left(n^{-3 / 4}\right) M^{2}\left(\xi_{n, 0}\right)+ \\
\quad\left(1+O\left(\frac{\log ^{2} n}{\sqrt{n}}\right)\right) \sum_{t=1}^{\infty} t \exp \left(-\frac{\pi t r}{\sqrt{6 n}}\right) \cdot \frac{\exp \left(-\frac{\pi t \Lambda^{\prime} d}{\sqrt{6 n}}\right)}{1-\exp \left(-\frac{\pi t d}{\sqrt{6 n}}\right)} .
\end{aligned}
$$

Using again the inequalities $\frac{1}{\beta}+\frac{1}{2}<\frac{1}{1-e^{-\beta}}<\frac{1}{\beta}+1$ (for $\beta>0$ ) and the formula $\sum_{t=1}^{\infty} t e^{-a t}=\frac{1}{\left(e^{a}-1\right)\left(1-e^{-a}\right)}$ we obtain

$$
\begin{aligned}
& D^{2}\left(\xi_{n, 0}\right) \leqq O\left(n^{-3 / 4}\right) M^{2}\left(\xi_{n, 0}\right)+ \\
& \quad+\left(1+O\left(\frac{\log ^{2} n}{\sqrt{n}}\right)\right) \sum_{t=1}^{\infty} t \exp \left(-\frac{\pi t\left(r+\Lambda^{\prime} d\right)}{\sqrt{6 n}}\right)\left\{\frac{\sqrt{6 n}}{\pi t d}+1\right\}= \\
& =O\left(n^{-3 / 4}\right) M^{2}\left(\xi_{n, 0}\right)+ \\
& \quad+\left(1+O\left(\frac{\log ^{2} n}{\sqrt{n}}\right)\right)\left\{\frac{\sqrt{6 n}}{\pi d} \cdot \frac{1}{\exp \left(\frac{\pi\left(r+\Lambda^{\prime} d\right)}{\sqrt{6 n}}\right)-1}+\right.
\end{aligned}
$$




$$
\begin{aligned}
&\left.+\frac{1}{\exp \left(\frac{\pi\left(r+\Lambda^{\prime} d\right)}{\sqrt{6 n}}\right)-1} \cdot \frac{1}{1-\exp \left(-\frac{\pi\left(r+\Lambda^{\prime} d\right)}{\sqrt{6 n}}\right)}\right\}= \\
&=\frac{1+O\left(\frac{\log ^{2} n}{\sqrt{n}}\right)}{\exp \left(\frac{\pi\left(r+\Lambda^{\prime} d\right)}{\sqrt{6 n}}\right)-1}\left\{\frac{\sqrt{6 n}}{\pi d}+\frac{1}{1-\exp \left(-\frac{\pi\left(r+\Lambda^{\prime} d\right)}{\sqrt{6 n}}\right)}+\right. \\
&\left.+\left(\sqrt{\exp \left(\frac{\pi\left(r+\Lambda^{\prime} d\right)}{\sqrt{6 n}}\right)-1} \cdot n^{-3 / 8}\left|M\left(\xi_{n, 0}\right)\right|\right)^{2}\right\} .
\end{aligned}
$$

Let $y \doteq \sqrt{\exp \left(\frac{\pi\left(r+\Lambda^{\prime} d\right)}{\sqrt{6 n}}\right)-1}$. We have

$$
\exp \left(\frac{\pi\left(r+\left(\Lambda^{\prime}+1\right) d\right)}{\sqrt{6 n}}\right)=\left(y^{2}+1\right) \exp \left(\frac{\pi d}{\sqrt{6 n}}\right) \geqq y^{2}+1
$$

and, by Lemma 1,

$$
\begin{gathered}
y \cdot n^{-3 / 8}\left|M\left(\xi_{n, 0}\right)\right|=O\left(y n^{-3 / 8}\right)\left\{\frac{\sqrt{n}}{d} \log \left(1+\frac{1}{y^{2}}\right)+\frac{1}{y^{2}}\right\}= \\
=O\left(n^{-3 / 8}\right)\left\{\frac{\sqrt{n}}{d} y \log \left(1+\frac{1}{y^{2}}\right)+\frac{1}{y}\right\} . \\
y \log \left(1+\frac{1}{y^{2}}\right) \leq\left\{\begin{array}{c}
y \cdot \frac{1}{y^{2}}=\frac{1}{y} \leqq 1 \\
y \log \frac{2}{y^{2}}=y \log 2+2 y \log \frac{1}{y} \leqq \\
\leqq y \log 2+2 y \frac{1}{y} \leqq \log 2+2 \quad \text { if } 0<y<1 .
\end{array}\right.
\end{gathered}
$$

Thus,

$$
\begin{aligned}
& \left(y \cdot n^{-3 / 8}\left|M\left(\xi_{n, 0}\right)\right|\right)^{2}=O\left(n^{-3 / 4}\right)\left\{\frac{\sqrt{n}}{d}+\frac{1}{y}\right\}^{2}= \\
& =O\left(n^{-3 / 4}\right)\left\{\frac{\sqrt{n}}{d}+n^{1 / 4}\right\}^{2}
\end{aligned}
$$

since $y>\sqrt{\frac{\pi\left(r+\Lambda^{\prime} d\right)}{\sqrt{6 n}}} \geqq \sqrt{\frac{\pi}{\sqrt{6}}} n^{-1 / 4}$. As $d \leqq \sqrt{n}$, we have

$$
\begin{aligned}
& O\left(n^{-3 / 4}\right)\left\{\frac{\sqrt{n}}{d}+n^{1 / 4}\right\}^{2}=O\left(n^{-3 / 4}\right)\left\{\frac{n}{d^{2}}+\frac{2 n^{3 / 4}}{d}+n^{1 / 2}\right\}= \\
& =O\left(n^{-3 / 4}\right) \frac{\sqrt{n}}{d}\left\{\frac{\sqrt{n}}{d}+2 n^{1 / 4}+d\right\}=O\left(n^{-3 / 4}\right) \frac{\sqrt{n}}{d}\left\{\sqrt{n}+2 n^{1 / 4}+\sqrt{n}\right\}= \\
& =\frac{\sqrt{n}}{d} O\left(n^{-1 / 4}\right) .
\end{aligned}
$$

Finally,

$$
D^{2}\left(\xi_{n, 0}\right) \leqq
$$




$$
\begin{aligned}
& \leqq \frac{1+O\left(n^{-1 / 4}\right)}{\exp \left(\frac{\pi\left(r+\Lambda^{\prime} d\right)}{\sqrt{6 n}}\right)-1}\left\{\frac{\sqrt{6 n}}{\pi d}+\frac{1}{1-\exp \left(-\frac{\pi\left(r+\Lambda^{\prime} d\right)}{\sqrt{6 n}}\right)}\right\}= \\
& =\frac{O(1)}{\exp \left(\frac{\pi\left(r+\Lambda^{\prime} d\right)}{\sqrt{6 n}}\right)-1}\left\{\frac{\sqrt{n}}{d}+1+\frac{1}{\exp \left(\frac{\pi\left(r+\Lambda^{\prime} d\right)}{\sqrt{6 n}}\right)-1}\right\}= \\
& =\frac{O(1)}{\exp \left(\frac{\pi\left(r+\Lambda^{\prime} d\right)}{\sqrt{6 n}}\right)-1}\left\{\frac{\sqrt{n}}{d}+\frac{\sqrt{n}}{r+\Lambda^{\prime} d}\right\}= \\
& =\frac{O(1)}{\exp \left(\frac{\pi\left(r+\Lambda^{\prime} d\right)}{\sqrt{6 n}}\right)-1} \cdot \frac{\sqrt{n}}{\min \left(d, r+\Lambda^{\prime} d\right)} .
\end{aligned}
$$

$[\alpha=1:]$

For $\Lambda \leqq \frac{\sqrt{6}}{\pi} \sqrt{n} \log n$ and $d \leqq \sqrt{n}$, using Lemma 1 and Remark 1 ,

$$
\begin{aligned}
& D^{2}\left(\xi_{n, 1}\right) \leqq O\left(n^{-3 / 4}\right) M^{2}\left(\xi_{n, 1}\right)+ \\
& \quad+\left(1+O\left(\frac{\log ^{2} n}{\sqrt{n}}\right)\right) \sum_{\substack{k \geq \Lambda^{\prime} \\
t \geq 1 \\
t(r+k d)<s}}(r+k d)^{2} t \exp \left(-\frac{\pi t(r+k d)}{\sqrt{6 n}}\right) \leqq \\
& \leqq O\left(n^{-3 / 4}\right) M^{2}\left(\xi_{n, 1}\right)+
\end{aligned}
$$$$
+O\left(s^{2}\right) \quad \sum_{k \geq \Lambda^{\prime}} \frac{1}{t} \exp \left(-\frac{\pi t(r+k d)}{\sqrt{6 n}}\right) \leqq
$$$$
t(r+\underline{t} d)<s
$$$$
\leqq O\left(n^{-3 / 4}\right)\left\{\frac{n}{d}+\left(r+\Lambda^{\prime} d\right) \frac{\sqrt{n}}{d} \log n+\sqrt{n} d\right\}^{2}+
$$$$
+O\left(n \log ^{2} n\right) \sum_{t=1}^{\infty} \frac{1}{t} \exp \left(-\frac{\pi t r}{\sqrt{6 n}}\right) \sum_{k=\Lambda^{\prime}}^{\infty} \exp \left(-\frac{\pi t d k}{\sqrt{6 n}}\right) \leqq
$$$$
\leqq O\left(n^{-3 / 4}\right)\left\{\frac{n}{d}+(\Lambda+d) \frac{\sqrt{n}}{d} \log n+\sqrt{n} d\right\}^{2}+
$$$$
+O\left(n \log ^{2} n\right) \sum_{t=1}^{\infty} \frac{1}{t} \exp \left(-\frac{\pi t\left(r+\Lambda^{\prime} d\right)}{\sqrt{6 n}}\right) \frac{1}{1-\exp \left(-\frac{\pi t d}{\sqrt{6 n}}\right)} \leqq
$$$$
\leqq O\left(n^{-3 / 4}\right)\left\{\frac{n \log ^{2} n}{d}+\sqrt{n} d\right\}^{2}+
$$$$
+O\left(n \log ^{2} n\right) \sum_{t=1}^{\infty} \frac{1}{t} \exp \left(-\frac{\pi t\left(r+\Lambda^{\prime} d\right)}{\sqrt{6 n}}\right)\left\{\frac{\sqrt{6 n}}{\pi t d}+O(1)\right\} \leqq
$$$$
\leqq O\left(n^{1 / 4}\right)\left\{\frac{\sqrt{n} \log ^{2} n}{d}+d\right\}^{2}+
$$$$
+O\left(n \log ^{2} n\right)\left\{\frac{\sqrt{6 n}}{\pi d} \sum_{t=1}^{\infty} \frac{1}{t^{2}}+O\left(\log \frac{1}{1-\exp \left(-\frac{\pi\left(r+\Lambda^{\prime} d\right)}{\sqrt{6 n}}\right)}\right)\right\}=
$$ 


$$
\begin{aligned}
& =O\left(n^{1 / 4}\right)\left\{\frac{\sqrt{n} \log ^{2} n}{d}+d\right\}^{2}+O\left(n \log ^{2} n\right)\left\{\frac{\sqrt{n}}{d}+\log n\right\}= \\
& =O(1)\left\{\frac{n^{5 / 4} \log ^{4} n}{d^{2}}+n^{3 / 4} \log ^{2} n+n^{1 / 4} d^{2}+\frac{n^{3 / 2} \log ^{2} n}{d}+n \log ^{3} n\right\}= \\
& =O(1)\left\{\frac{n^{3 / 2} \log ^{2} n}{d}+n \log ^{3} n+n^{1 / 4} d^{2}\right\}= \\
& =O\left(\frac{n^{3 / 2}}{d}\right)\left\{\log ^{2} n+\frac{d \log ^{3} n}{\sqrt{n}}+\frac{d^{3}}{n^{5 / 4}}\right\} .
\end{aligned}
$$

For $d \leqq n^{5 / 12}$, we obtain that

$$
D^{2}\left(\xi_{n, 1}\right)=O\left(\frac{n^{3 / 2} \log ^{2} n}{d}\right) .
$$

\section{COMPLETIONS OF THE PROOFS OF THE THEOREMS}

Case $\alpha=0$. Lemmas 1 and 2 for $\alpha=0$ give the assertion in our Theorem 1 :

Let $\omega(n) \nearrow \infty, d \leqq \sqrt{n}, \Lambda \leqq \frac{\sqrt{6}}{\pi} \sqrt{n} \log n$. Applying Chebyshev's inequality and using Remark 2 , we obtain that, for all but $p(n) / \omega(n)$ partitions of $n$,

$$
\begin{aligned}
& \sum_{\substack{\mu \\
\lambda_{\mu} \equiv r(d) \\
\lambda_{\mu} \geqq \Lambda}} 1=M\left(\xi_{n, 0}\right)+O\left(\sqrt{\frac{\sqrt{n} \omega(n)}{\left(\exp \left(\frac{\pi\left(r+\Lambda^{\prime} d\right)}{\sqrt{6 n}}\right)-1\right) \min \left(d, r+\Lambda^{\prime} d\right)}}\right)= \\
& =\left(1+O\left(\frac{\log ^{2} n}{\sqrt{n}}\right)\right)\left\{\frac{\sqrt{6 n}}{\pi d} \log \frac{1}{1-\exp \left(-\frac{\pi\left(r+\left(\Lambda^{\prime}+1\right) d\right)}{\sqrt{6 n}}\right)}+\right. \\
& \left.+\frac{O\left(\frac{\exp \left(\frac{\pi\left(r+\Lambda^{\prime} d\right)}{\sqrt{6 n}}\right)-1}{\exp \left(\frac{\pi\left(r+\left(\Lambda^{\prime}+1\right) d\right)}{\sqrt{6 n}}\right)-1}+O\left(\sqrt{\frac{\sqrt{n} \omega(n)}{\left(\exp \left(\frac{\pi\left(r+\Lambda^{\prime} d\right)}{\sqrt{6 n}}\right)-1\right) \cdot \min \left(d, r+\Lambda^{\prime} d\right)}}\right)\right.}{\exp }\right)
\end{aligned}
$$

where $\Lambda^{\prime}=\left\lceil\frac{\Lambda-r}{d}\right\rceil, 1 \leqq r \leqq d$. Again with $y=\sqrt{\exp \left(\frac{\pi\left(r+\Lambda^{\prime} d\right)}{\sqrt{6 n}}\right)-1}$,

$$
\begin{aligned}
& O\left(\frac{\log ^{2} n}{\sqrt{n}}\right) \frac{\sqrt{6 n}}{\pi d} \log \left(1+\frac{1}{y^{2}}\right)+\frac{O(1)}{y^{2}}= \\
& =O\left(\frac{1}{y}\right)\left\{\frac{\log ^{2} n}{d} y \log \left(1+\frac{1}{y^{2}}\right)+\frac{1}{y}\right\} \leqq \\
& \leqq O\left(\frac{1}{y}\right)\left\{\frac{\log ^{2} n}{d} O(1)+O(1) \sqrt{\frac{\sqrt{n}}{r+\Lambda^{\prime} d}}\right\}=O\left(\frac{1}{y}\right) \sqrt{\frac{\sqrt{n}}{\min \left(d, r+\Lambda^{\prime} d\right)}}
\end{aligned}
$$

thus our Theorem 1 is proved. 
Case $\alpha=1$. Lemmas 1 and 2 for $\alpha=1$ give the assertion in our Theorem 2 :

Let $\omega(n) \nearrow \infty, d \leqq n^{5 / 12}, \Lambda \leqq \frac{\sqrt{6}}{\pi} \sqrt{n} \log n$. Applying Chebyshev's inequality and using Remark 1, we obtain that, for all but $p(n) / \omega(n)$ partitions of $n$,

$$
\begin{aligned}
& \sum_{\substack{\mu \\
\lambda_{\mu} \equiv r(d) \\
\lambda_{\mu} \geqq \Lambda}} \lambda_{\mu}= \\
& =M\left(\xi_{n, 1}\right)+O\left(\frac{n^{3 / 4}}{\sqrt{d}}(\log n) \sqrt{\omega(n)}\right)= \\
& =\left(1+O\left(\frac{\log ^{2} n}{\sqrt{n}}\right)\right)\left\{\frac{n}{d}-\frac{6 n}{\pi^{2} d} \sum_{t=1}^{\infty} \frac{1}{t^{2}}\left(1-\exp \left(-\frac{\pi\left(r+\Lambda^{\prime} d\right) t}{\sqrt{6 n}}\right)\right)+\right. \\
& \left.+\left(r+\Lambda^{\prime} d\right) \frac{\sqrt{6 n}}{\pi d} \log \frac{1}{1-\exp \left(-\frac{\pi\left(r+\Lambda^{\prime} d\right)}{\sqrt{6 n}}\right)}+\frac{O\left(\left(\Lambda^{\prime}+1\right) d\right)}{\exp \left(\frac{\pi\left(r+\Lambda^{\prime} d\right)}{\sqrt{6 n}}\right)-1}\right\}+ \\
& +O\left(\frac{n^{3 / 4} \log n}{\sqrt{d}} \sqrt{\omega(n)}\right)= \\
& =\left(1+O\left(\frac{\log ^{2} n}{\sqrt{n}}\right)\right)\left\{\frac{n}{d}+O\left(\frac{\sqrt{n}}{d}\left(r+\Lambda^{\prime} d\right) \log n\right)+O\left(\frac{1}{d}\right)+\right. \\
& \left.+O\left(\left(r+\Lambda^{\prime} d\right) \frac{\sqrt{n}}{d} \log n\right)+O(\sqrt{n} d)\right\}+ \\
& +O\left(\frac{n^{3 / 4} \log n}{\sqrt{d}} \sqrt{\omega(n)}\right)= \\
& =\left(1+O\left(\frac{\log ^{2} n}{\sqrt{n}}\right)\right)\left\{\frac{n}{d}+O\left(\frac{\sqrt{n}}{d}\left(r+\Lambda^{\prime} d\right) \log n\right)+O(\sqrt{n} d)\right\}+ \\
& +O\left(\frac{n^{3 / 4} \log n}{\sqrt{d}} \sqrt{\omega(n)}\right)= \\
& =\frac{n}{d}+O\left(\frac{\sqrt{n}}{d} \log ^{2} n\right)+O\left(\frac{\sqrt{n}}{d}\left(r+\Lambda^{\prime} d\right) \log n\right)+O(\sqrt{n} d)+ \\
& +O\left(\frac{n^{3 / 4} \log n}{\sqrt{d}} \sqrt{\omega(n)}\right) \text {. }
\end{aligned}
$$

For $d \leqq n^{1 / 6} \sqrt{n} d \leqq \frac{n^{3 / 4}}{\sqrt{d}}$ so that we get

$$
\frac{n}{d}+O\left(\frac{\sqrt{n}}{d}\left(r+\Lambda^{\prime} d\right) \log n\right)+O\left(\frac{n^{3 / 4} \log n}{\sqrt{d}} \sqrt{\omega(n)}\right)
$$

which completes the proof of Theorem 2 .

\section{REFERENCES}

[1] C. Dartyge and A. Sárközy, Arithmetic properties of summands of partitions, to appear in Ramanujan $J$.

[2] C. Dartyge and A. Sárközy, Arithmetic properties of summands of partitions, II, to appear in Ramanujan J. 
[3] P. Erdős and J. Lehner, The distribution of the number of summands in the partitions of a positive integer, Duke Math. Journal, 8 (1941), 335-345.

[4] P. Erdős and M. Szalay, Note to Turán's papers on the statistical theory of groups and partitions, in: Collected Papers of Paul Turán, Akadémiai Kiadó, Budapest, 1990, Vol. 3, 2583-2603.

[5] G. H. Hardy and S. Ramanujan, Asymptotic formulae in combinatory analysis, Proc. London Math. Soc., 17 (1918), 75-115.

[6] M. Szalay and P. Turán, On some problems of the statistical theory of partitions with application to characters of the symmetric group III, Acta Math. Acad. Sci. Hungar., 32 (1978), 129-155.

Cécile Dartyge

Institut Élie Cartan

Université Henri Poincaré - Nancy 1

BP 239

54506 Vandoeuvre Cedex

France

dartyge@iecn.u-nancy.fr

András Sárközy and Mihály Szalay

Department of Algebra and Number Theory

Eötvös Loránd University

H-1518 Budapest, Pf. 120

H-1117 Budapest

Pázmány Péter sétány 1/C

Hungary

sarkozy@cs.elte.hu

mszalay@cs.elte.hu 